\title{
Preoperative tomographic assessment of the mediastinum in bronchial carcinoma
}

\author{
P. K. PEACE and J. L. PRICE
}

Milford Chest Hospital, Surrey

The operation findings in 97 patients with histologically proven bronchial carcinoma are correlated with inclined frontal tomograms in order to assess mediastinal lymphadenopathy. The diagnostic accuracy of inclined frontal tomography is analysed.

Inclined frontal tomography was introduced into Milford Chest Hospital in 1969 and since that time has been in routine use for the investigation of the mediastinum and for assessing thoracic lymph node metastases in patients with bronchial carcinoma. This paper reports on the correlation of the radiological with the operative findings in 97 patients with histologically proven bronchial carcinoma during 1969 and 1970 and analyses the diagnostic accuracy of the technique.

In 1971 it was found that fewer patients were being subjected to palliative pneumonectomy with incomplete resection of malignant tumour and to exploratory thoracotomy. This was thought to be due to the influence of inclined frontal tomography in assessing operability. This was noted particularly in patients whose general condition permitted only a lobectomy and in patients with enlarged paratracheal and subcarinal lymph nodes which were considered to be too extensive for adequate surgical resection. In view of this, further correlation was not undertaken for this series.

\section{TECHNIQUE}

The first publication in English describing inclined frontal tomography was by Markovits and Desprez-Curely in 1962. Greenwell and Wright (1965) gave a full historical review and description of the method. We use the Massiot-Phillips radiotome as described in the paper by Greenwell and Wright but with modifications for determining the angle of cut on the preliminary radiograph and for reproducing the depth of cut on subsequent tomographic examination at a later date (Boyington, 1972). Similar results are obtained on a Siemens multiplanigraph by angulation of the film plane on a modified film tray and using a circular movement of the $x$-ray tube.

\section{DIAGNOSTIC CRITERIA}

A normal inclined frontal tomogram is shown in Figure 1. The trachea, main bronchi, and the main upper and lower lobe branches can be identified and the thickness of their walls determined. The apical segmental bronchi of the lower lobes and the middle lobe branches are at right angles to the plane of section and thus cannot be demonstrated with this technique. The aorta, pulmonary arteries, and azygos vein are prominent landmarks and the

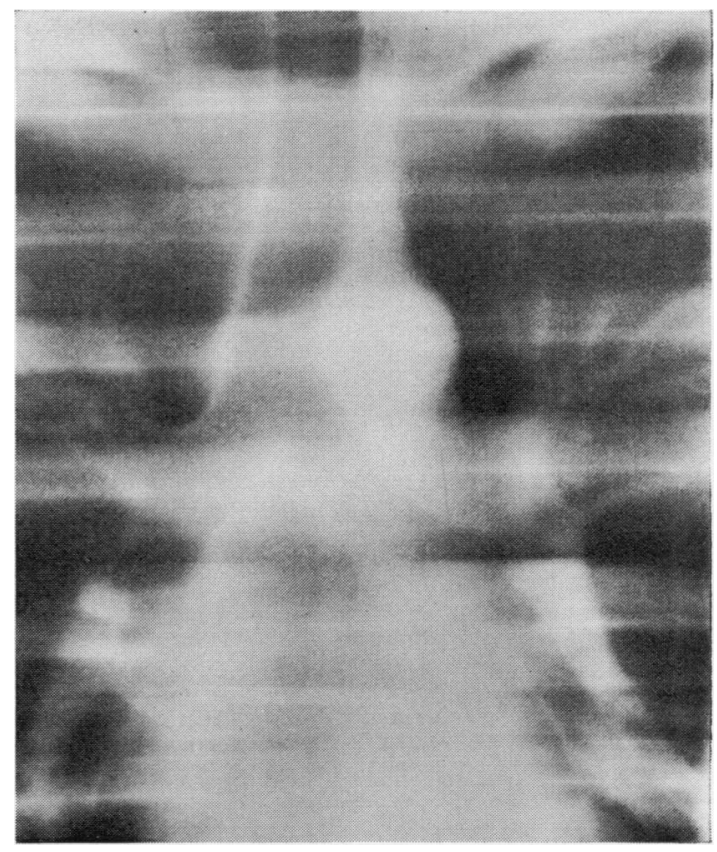

FIG. 1. Inclined plane tomogram in the plane of trachea and main bronchi showing normal anatomical structures. 
posterior pleural reflection of the lower mediastinum may be seen. Lymph nodes cannot be identified on the tomograms of a normal subject and in this study any lymph nodes seen on the tomograms were regarded as enlarged and pathological.

The enlarged lymph nodes were classified as paratracheal, tracheobronchial, and bronchopulmonary, but when seen the pretracheal, subcarinal, and subaortic nodes were described separately. Figure 2 demonstrates enlarged right bronchopulmonary and right para-aortic nodes due to metastases from a peripheral bronchial carcinoma. The pretracheal and subcarinal nodes are found on more anterior cuts and it is our practice to obtain at least five tomographic cuts to build up a composite picture of the mediastinal structures. The subaortic nodes are difficult to identify but lie on a posterior plane and tend to obliterate the notch between the aorta and the left main pulmonary artery. Figure 3 illustrates enlargement of subcarinal and subaortic nodes. Where possible an attempt is made to show the primary carcinoma on the tomogram but we agree with Greenwell and Wright (1965) that the principal value of this technique is in demonstrat-

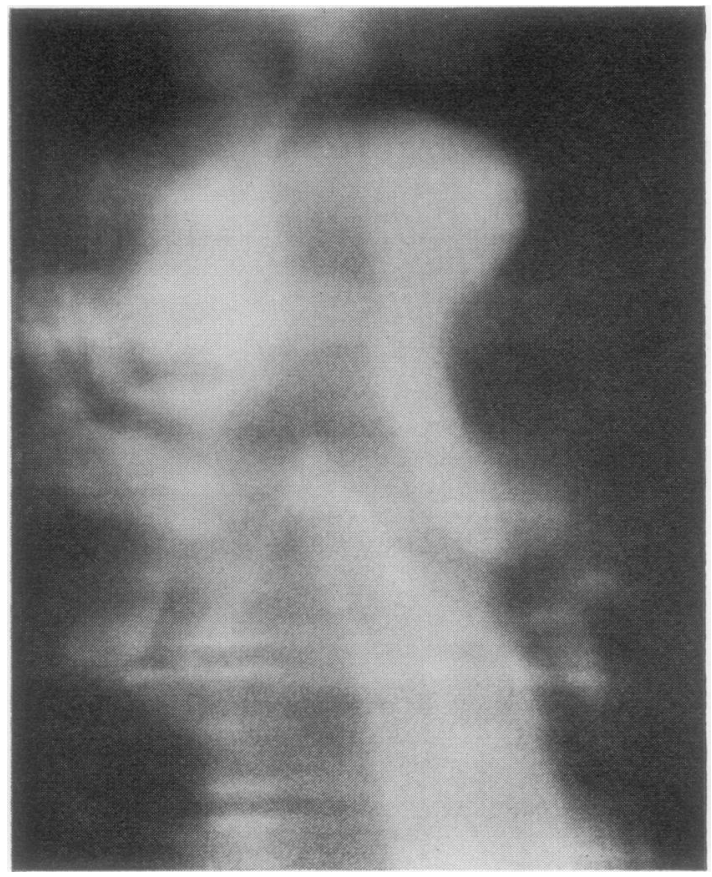

FIG. 2. Enlarged right bronchopulmonary nodes and right paratracheal nodes. Note the thickening of the walls of the right upper lobe bronchus.

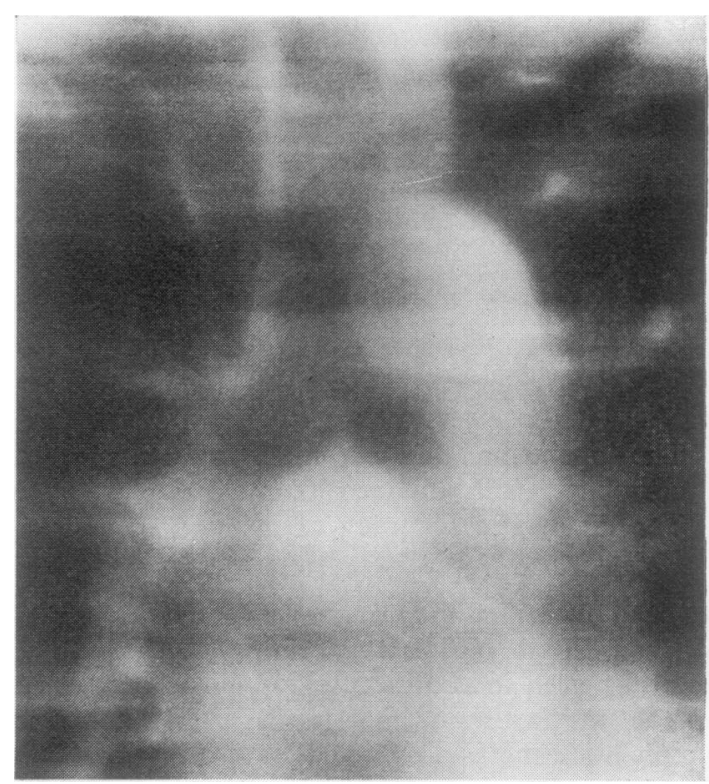

FIG. 3. A patient with carcinoma of the left lower lobe bronchus. A large subcarinal node is present and the notch between the aorta and left main pulmonary artery is obliterated, indicating involved subaortic nodes.

ing the trachea, main bronchi, and mediastinum and not a peripheral primary tumour.

\section{CLINICAL MATERIAL-METHOD}

During the period under review over 150 inclined frontal tomograms were performed, but only in 97 of these were the full operative findings and histology known. A proportion of the patients without full operative findings had tomograms for conditions other than bronchial carcinoma and some patients with carcinoma were judged inoperable on clinical, bronchoscopic or mediastinoscopic evidence.

The operation findings were recorded and these were then compared with the radiological findings $\mathcal{N}$ taken from the original reports without reviewing the films.

\section{RESULTS (See Table)}

LYMPHADENOPATHY PRESENT AT OPERATION The correlation between the operative findings and the ? tomographic demonstration of the enlarged metastatic lymph nodes was absolute in 42 cases. The $\frac{\vec{D}}{\mathbb{D}}$ eight patients in the group with good correlation $\stackrel{?}{\square}$ all had a shadow in the hilum but we were unable $\varnothing$ to distinguish the tumour and glands on the radiograph. The reports in these cases described hilar $O$ shadows and no attempt was made to distinguish 
T A B L E

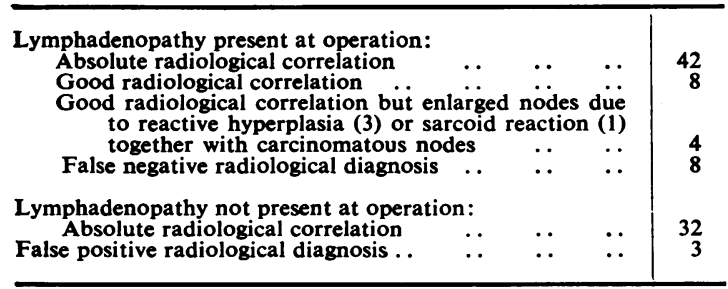

between tumour extension and lymphadenopathy; however, the extent of the shadow on the tomograms corresponded well with the extent of the tumour mass found at operation.

Three patients were found to have enlarged benign bronchopulmonary or subcarinal lymph nodes at operation and these had been identified on the tomograms. These nodes did not contain tumour on histological examination which showed reactive hyperplasia. These were all under $3 \mathrm{~cm}$ in diameter and it was considered that even if they had been malignant the tomographic appearances would not have deterred the surgeon from thoracotomy or even influenced the extent of the operation. One patient with bronchial carcinoma had metastatic involvement of a subcarinal node, but an enlarged bronchopulmonary node was also present and this was found to be due to a sarcoid reaction on histological examination. Both these glands were identified on the tomograms.

A false negative diagnosis was made in eight patients. Nodes missed on the tomograms are usually small and in five of these patients the primary tumour and the involved nodes were removed at operation.

Two patients had a radical pneumonectomy and three a lobectomy. In two of this group of patients, subaortic nodes were found at operation; in the remaining three patients, one was found to have a pathological subcarinal node, one a bronchopulmonary node, and one a pretracheal node. These nodes were not seen on the tomograms and histological examination confirmed that they were invaded by metastatic carcinoma. The abnormal tomographic appearances were misinterpreted in the three patients who were considered to be inoperable at thoracotomy. Two of these had extensive hilar masses and one also had subcarinal gland involvement. One patient had a $5 \mathrm{~cm}$ mediastinal mass involving the phrenic nerve.

LYMPHADENOPATHY NOT PRESENT AT OPERATION In the group in which there was absolute correlation between the surgical and tomographic findings, eight patients had nodes removed which were thought to be suspect by the surgeon but on histological examination were found to be free of metastatic deposits. In 24 patients there were no suspect nodes at thoracotomy. The suspected but histologically normal nodes were all small and the largest measured $2 \mathrm{~cm}$ in diameter. None of these nodes was seen on the tomogram and although nodes measuring less than $2 \mathrm{~cm}$ in diameter may sometimes be identified on the film when invaded by metastatic carcinoma, this is probably due to the increased radiographic density of the tumour material and the associated fibrotic changes.

The false positive diagnoses are more dangerous because a surgeon may be dissuaded from operating on patients in whom the carcinoma is resectable. The three false positives in this series where the interpretation of the tomograms was misleading are considered in more detail.

Case 1 A 56-year-old man had a bronchoscopically proved squamous-cell carcinoma of the right upper lobe bronchus. The radiological findings were obliteration of the right upper lobe bronchus and some

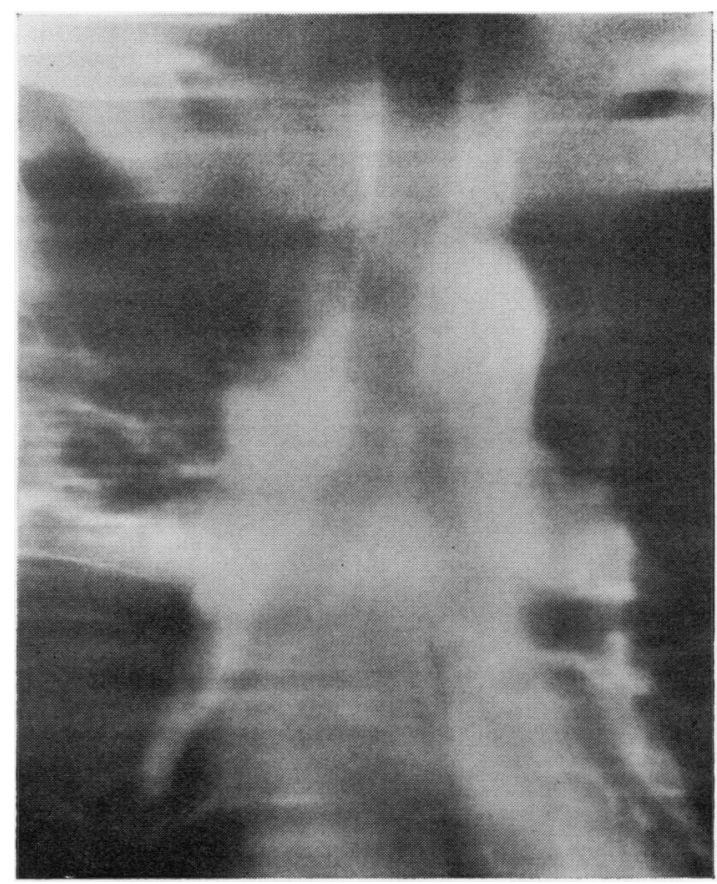

FIG. 4. Case 1. The films show obliteration of the right upper lobe bronchus with a surrounding mass extending into the azygos vein. This was misinterpreted as right lower paratracheal lymph node involvement. 
irregularity of the upper wall of the right main bronchus. A density was apparent to the right of the trachea in the region of the azygos vein with thickening of the tracheal wall, and this was thought to be an enlarged paratracheal lymph node. An enlarged pretracheal gland was also suspected (Fig. 4). At operation the tumour was found to be the size of a walnut and was adherent to the azygos vein and superior vena cava and a radical pneumonectomy was performed. Although some bronchopulmonary nodes were involved there was no evidence of paratracheal or pretracheal lymphadenopathy. In retrospect there is good correlation between the tomograms and the operative findings and this emphasizes the difficulties in differentiating between direct tumour extension and lymph node enlargement, particularly when the tumour mass extends forwards to the superior vena cava. Dense anterior masses may also cause confusing sweep shadows in the pretracheal region.

Case 2 A 66-year-old man had a squamous-cell carcinoma of the right upper lobe. The tomogram report suggested that the right wall of the trachea was thickened and merged with the tumour mass, and from this it was inferred that paratracheal lymphadenopathy was present. At operation the tumour was found to be extending medially and was adherent to the parietal pleura but not invading it, but no enlarged or metastatic paratracheal nodes were found. An upper lobectomy with sleeve resection was performed.

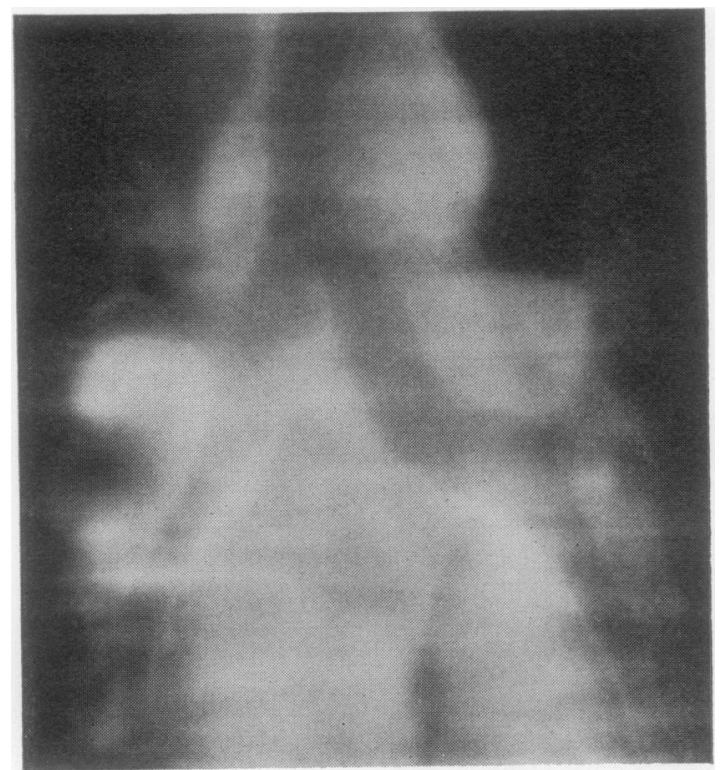

FIG. 5. Case 3. The tomogram demonstrates enlargement of a right paratracheal node. Irregularity of the medial margin of the right main bronchus can be seen. This was interpreted as involvement of the bronchial wall by a metastatic subcarinal node, but was proved to be tumour infiltration of the mucosa at operation.
Case 3 A 58-year-old man was found to have a right upper lobe lesion on a routine chest film. At bronchoscopy a right upper lobe epidermoid carcinoma was found and tumour infiltration of the mucosa of the lower border of the right main bronchus was discovered. A right pneumonectomy was performed. An enlarged malignant right paratracheal node was removed but no other pathological nodes were found. This node had been visible on the tomograms and the irregularity of the lower wall of the right main bronchus was also described. An enlarged subcarinal node had been reported on the tomograms lying ad- $\times$ jacent to the right main bronchus but in retrospect the angle of the tracheal bifurcation was too narrow to $\omega$ contain a significant node (Fig. 5).

\section{CONCLUSIONS}

The diagnostic accuracy of inclined frontal tomograms when compared with the findings at thoracotomy is high. The finding that enlarged $v$ lymph nodes are absent on tomography can be $\omega$ accepted with confidence. Difficulty may be experienced in differentiating hilar node masses from the primary tumour and caution is needed $\bar{\partial}$ in interpreting upper lobe masses lying adjacent to the mediastinum as these may not be invading $\varrho$ the paratracheal structures. In terms of the $\overrightarrow{\vec{B}}$ anatomical site of the neoplastic process the tomo- $\frac{0}{3}$ grams are remarkably accurate. In assessing operability the investigation is complementary to bronchoscopy and the results are encouraging. In the group of 51 patients with lung carcinoma음 where lymphadenopathy was confirmed at oper- $\frac{0}{x}$ ation, 16 patients had surgery limited to explora- $\dot{\sigma}$ tory thoracotomy, and six a palliative pneumon. ectomy. In the group of 32 patients with no lymph-ᄋ adenopathy, in only one patient was the tumour not resected and this was due to neoplastic inva-을 sion of the chest wall. We have found that this technique facilitates the selection of patients for을. mediastinoscopy and is also a useful aid in plan- $N$ ning radiotherapy.

The patients in this series were under the care of Dr. F. E. Joules, Mr. M. Meredith Brown, Mr. V. Powell, and Mr. R. Rowlandson and we are grateful to them for their advice and encouragement in the preparation $\frac{C}{\mathscr{D}}$ of this paper.

\section{REFERENCES}

Boyington, P. (1972). The radiotome. Radiography, 38, 137. Greenwell, F. P., and Wright, F. W. (1965). RotationalQ tomography. Clin. Radiol., 16, 377.

Markovits, P., and Desprez-Curely, J. P. (1962). Inclined frontal tomography in the examination of the media-O stinum. Radiology, 78, 371 . 